\title{
The Collapse of Sensemaking in Injury Root Cause Investigations Resulting in Ineffective Injury Prevention Decision-Making: A Retrospective Case Study
}

\author{
Anton Shufutinsky ${ }^{1 *}$, Ryan $\operatorname{Cox}^{2}$ and Maria E Vizcarrondo ${ }^{3}$ \\ ${ }^{1}$ Academy of Interdisciplinary Health Science Leaders, Changineering Global, USA \\ ${ }^{2}$ Honor Society for International Scholars, USA \\ ${ }^{3}$ Nerney Leadership Institute, USA
}

Submission: June 13, 2017; Published: June 28, 2017

*Corresponding author: Anton Shufutinsky, Academy of Interdisciplinary Health Science Leaders, Changineering Global, PA 19027, USA,

Email: antonshufu@icloud.com

\begin{abstract}
One of the main tasks of workplace public health professionals is management of injury reduction programs. A critical part of managing in complex environments is effective Sensemaking. Sensemaking is a critical leadership skill necessary in order to determine what has occurred or is occurring in an organization or a given situation, and a means of identifying ways to improve on the results or prevent ramifications. In the industrial setting, environmental health and safety organizations must understand the importance of sensemaking as a critical skill for EHS leaders to perform adequate and accurate injury investigations and prevent recurrence of workplace casualties. Root cause investigations must include a strategy capable of identifying the equipment, policies, environment, and behaviors that caused or contributed to workplace injuries. In this retrospective case study, we evaluated a flawed root cause investigation and leadership decisions through a sense making lens and determined that a collapse in sensemaking resulted in poor decision-making, leading to an incorrect root cause and an inability to assign adequate corrective and preventive actions to inhibit recurrence of similar incidents.
\end{abstract}

Keywords: Root cause analysis; Accident investigation; Sensemaking; Organizational development; Occupational safety; Cognitive bias; Injury prevention

\section{Introduction}

One of the main tasks of workplace public health professionals is management of injury reduction programs. A critical part of managing in complex environments is effective sensemaking. Sensemaking is a critical leadership skill necessary in order to determine what has occurred or is occurring in an organization or a given situation, and a means of identifying ways to improve on the results or prevent ramifications. In the industrial setting, environmental health and safety organizations must understand the importance of sensemaking as a critical skill for EHS leaders to perform adequate and accurate injury investigation and prevent recurrence of workplace casualties. Root cause investigations must include a strategy capable of identifying the equipment, policies, environment, and behaviors that caused or contributed to workplace injuries. In this retrospective case study, we evaluated a flawed root cause investigation and leadership decisions through a sensemaking lens and determined that a collapse in sense making resulted in poor decision-making, leading to an incorrect root cause and an inability to assign adequate corrective and preventive actions to inhibit recurrence of similar incidents.

\section{Literature Review}

The Need for Root Cause Analysis Methods in Accident Investigations

Accident investigations must be thorough because causative agents for accidents in the workplace can result from poor

a. safety management systems,

b. engineering controls,

c. personal protective measures, or

d. Safety culture programs [1].

Determining individual and interacting factors that cause or contribute to injury is critical for future prevention efforts [1-4]. 
Incident root causes can vary, being driven by anything from

i. faulty safety management systems,

ii. inadequate personal protective equipment,

iii. machinery malfunction,

iv. improper engineering controls, or

v. Ineffective organizational culture. Determining all of these contributing factors and the specifics behind them that led to injury is vital in order to prevent similar occupational hazards in the future, and prevent recurrence of similar injuries [1-4].

RCA and similar models being used in industry today include

a) Accident Root Cause Tracing,

b) Fault Tree Analysis,

c) Barrier Analysis,

d) Ishikawa-Fishbone Cause and Effect, and

e) the 5-Why method, among others [1].

These methods are used to analyze whether the necessary barriers were in place to prevent the injury being investigated, as well as analyzing the
a. equipment,
b. work processes,
c. work environment,
d. behaviors, and

acts of Mother Nature, and how they contributed to the occurrence [1]. In essence, these tools are used to assist in making sense out of accidents during investigations. Sensemaking is inherently necessary in the root because investigation processes.

\section{Organizational Decision-Making}

Decision-making is critical in organizations because it drives the actions that are taken by those organizations and their leaders. These actions essentially determine organizational results and whether or not an establishment is successful with regard to the organizational mission and goals, including the provision of a safe work environment for employees. The natural human decision-making processes are unreliable, much because human beings are often influenced by many factors when in order to make decisions [5,6]. Inconsistent decision-making is a costly problem for many organizations, often resulting from individual human judgments that are influenced by cognitive biases and irrelevant factors called noise, including items such as personal mood and environmental weather, among others [6].

\section{Additional factors, such as}
I. Hermeneutics
II. emotions
III. social influences
IV. information availability and

\section{V. moral motivations}

play a pivotal role in cognitive bias and thus in decisionmaking [7]. Sensemaking, defined in short form as a constructed and coordinated system of action [7], evaluates and steers decision-making, including early and follow-on decisions after successful or failed actions. Effective sense making is critical for organizational success and for reducing potential losses generated by interference of noise and other factors.

\section{Sensemaking for Organizational Decisions}

Sensemaking is central to organizational decisions because it is where meanings materialize and inform or drive action $[7,8]$. It is the natural and ongoing process by which we investigate whether a decision for action was the right one, and the resultant effects of the action exercised [8]. This process can sometimes be easily taken for granted and, if not practiced carefully, may lead to a domino effect of like decisions, even if they are bad ones, because it is not unlikely that an individual's or organization's action establishes commitment.

The complex process of sense making is all about evaluating, from a comprehensive approach, what occurs in a given situation, and projecting how it can affect a particular situation and the involved organization. The ability to
a. analyze
b. interpret
c. search
d. view, and
e. think

about the situation is critical for effective decision-making. The common themes in sensemaking are psychodynamic in nature, often focusing on levels of awareness that impact sense making and the social biases and interpretations that ultimately influence organizational or individual action [9], and the results of those actions.

\section{Sensemaking and Root Cause Analysis}

In industrial settings, root cause investigations are performed for many purposes, including for identification of the cause of injuries and environmental incidents and the prevention of recurrence. Safety-driven root cause investigations are generally performed by environmental health and safety (EHS) professionals and EHS engineers. Although not clinical practitioners, these professionals are usually grouped in an occupational category of preventive health specialists, and are therefore health professionals. The idea of sensemaking is not a new concept in healthcare, although it has been somewhat overlooked in applied health management research. Davidson, Daly, [10], however, developed a sense making model for health care by combining the $[11,12]$ leadership organizational sensemaking model and [13] adaptation model. They created the Facilitated Sensemaking Model as an applied method 
provided by nursing staff to adjust to disruption experienced by families in situations where family members are in intensive care, allowing for better understanding, making sense of the situations they are newly exposed to, and decision-making Davidson, Daly, Agan, Brady, Higgins [10]. Such models would be useful for environmental health and safety root cause investigators, affording

- $\quad$ injured employees

- their supervisors

- their managers

- case managers

- quality assessors and

- $\quad$ senior leaders.

The ability to make sense of injuries and their causes, as well as necessary follow-on preventive measures. [8] loosely define sense making as an ongoing retrospective, organization, and ordering of all plausible cues or images in a situation in order to rationalize what people are doing. Sensemaking employs a multitude of texts, including analysis of narratives, to build actionable knowledge. The narrative is fundamental to sensemaking and leads to the enactment and retention of organizational action $[14,15]$. Through narratives, organizational actors are able to translate occurrences into meaningful events and thus impose a logical structure upon a flow of equivocal happenings through ordering and sequencing [14].

This is essentially the rationale behind root cause analysis, and [8], even if not purposefully, rudimentarily exhibits root cause analysis in a retrospective assessment of a collapse of sense making in a firefighting disaster that led to numerous casualties. This study contributed to the literature and knowledge regarding the necessity for certain skills and attributes necessary to foster effective decision-making in organizations. [8] go on to describe the nature of organized sense making from conceptual, descriptive, and prospective views, addressing
i. flux
ii. bracketing
iii. labeling
iv. retrospection
v. presumption
vi. plausibility
vii. identity
viii power and
ix emotion

as they apply to decision-making in organizations. [8] is credited with the greatest contributions in defining sense making, setting the stage for further research and thereby expanding the theoretical framework for analytical study of leadership and organizational decision-making.

\section{Materials and Methods}

\section{Reasoning}

There is limited literature on the application of sensemaking. Although there are plenty of articles that describe sensemaking and provide a theoretical basis for understanding the topic, a literature search yielded that although there are a number of articles that focus on sensemaking in healthcare settings, academia, and in professional service organizations [9,15-17], the peer-reviewed collection of manuscripts on applied sense making in niche fields, such as environmental health and safety, is limited or non-existent. This leaves room for research focused on the application and exploration of sensemaking in these real world organizational environments.

\section{Methods}

Using a phenomenological grounded theory approach, we performed a qualitative-inductive, naturalistic retrospective analysis of a case study involving a root cause investigation in a large corporate setting. The investigative data collection was conducted in real-time using a partially embedded ethnographic approach through ideographic, observation discovery and data analysis occurring retrospectively against the incident narrative through a sensemaking lens. Sensemaking was analyzed using definitions established in the peer-reviewed literature [7,9,15,18-21].

\section{Narrative of the Scenario}

At a materials manufacturing plant, a medium-sized occupational safety department of ten staff members serve as investigators for occupational injuries and illness. Incident root cause investigations. Investigations for all accidents and near misses are conducted by trained and experienced field investigators. Investigations for incidents that result in recordable injuries (RI) and lost-time injuries (LTI) are further reviewed and briefed by senior corporate leaders routinely, including senior compliance leaders, as they can become legal documentation in the event of regulatory inquiry.

Employees at a warehouse for the large manufacturing organization generally move a large variety of products from the warehouse to the employees throughout numerous buildings. These products vary, and are general items delivered for manufacturing and construction of final products that the corporation places on the market. The sizes and weights of materials moved and delivered vary, and may include large boxes, large solid components, machine parts, and drums of chemical compounds. Materials are generally moved and delivered on carts, with dollies, or via forklift. Some items must be stored or handled in the warehouse for short-term or long-term periods. Moving the large, awkward, or excessively heavy objects onto or off of pallets, onto forklifts, or onto or off of storage racks routinely require the assistance of moveable-arm vacuumassisted hoists in order to prevent musculoskeletal injuries to warehouse employees. 
During normal daily operations, an employee in a warehouse applied a mounted moveable-arm vacuum hoist in order to lift a 50 gallon drum of a cement compound necessary for a manufacturing process of a corporate product. The drum weighed approximately 350 pounds, requiring the vacuum hoist to move the drum from the storage pallet onto a drum shaker that is located 30 inches above floor level. The drum shaker is routinely used in order to shake the drum to loosen/soften the cement mixture compound if it is hardened upon receipt. Once the drum of compound is shaken for 15 minutes, it is lowered back onto the pallet using the vacuum hoist before it is transferred to the manufacturing location by fork truck.

After lowering a drum of shaken cement compound back onto a pallet, an employee was unable to remove the vacuum hoist from the drum. Apparently, the head plate, which is under vacuum seal, was not releasing because the release mechanism normally used to remove the seal was not functioning properly. Because of thus unanticipated problem, the employee was not able to remove the hoist from the drum, slowing delivery of the needed compound. In an attempt to rectify the situation, the employee called a co-worker over for assistance. Together, they decided that the co-worker would hold the top of the drum and push downward on it while the hoist operator attempted to apply excess pressure to the release mechanism and simultaneously pull upwards in order to release the hoist. After a few attempts, the seal was broken. However, the combination of the force with the rapid release caused the released head plate to immediately projected upwards. The co-worker who was holding the drum down was in a leaning position over the top of the drum. The unexpected and rapid upward retreat of the moveable arm caused the head plate to strike the employee in the head, requiring that the worker be evacuated to a local hospital emergency room for medical treatment.

\section{Root cause investigation results}

A thorough root cause investigation was performed utilizing four commonly-used root cause analysis tools, including Barrier Analysis, Fault-Tree Analysis, Ishikawa-Fishbone Cause and Effect Model, and the 5-Why method.

\section{Root and contributing causes}

The vacuum release mechanism malfunctioned. The process design, personnel actions, and equipment were assessed thoroughly, and it was discovered that there were numerous failures in the process that significantly contributed to the accident and injury. To begin with, there were no standard troubleshooting procedures established for equipment malfunction, despite the fact that this problem had been experienced on multiple occasions over several years. Troubleshooting should have been addressed through a managerial process and proceduralized, which is what would normally occur in the environment. Furthermore, although a procedure was not developed, there was no existing hands-on training provided to employees on how to correct the problem, despite the fact that this problem had been experienced on multiple occasions over several years.

Third, mechanical equipment, including fork trucks, carts, emergency showers, and many others are generally placed on a routine preventive maintenance schedule to ensure ideal function and operation. There was no established preventive maintenance schedule for the vacuum hoist and release mechanism and the equipment had not been inspected and maintained since the manufacturer's one-year warranty maintenance four years earlier. Finally, it was noted that the vacuum hoist is only used in this process when the cement material arrives in the warehouse in a hardened condition. Otherwise, the drum is delivered by forklift directly to the manufacturing area, without the need for the use of the hoist.

\section{Corrective and preventive actions}

Root cause investigations are critical for identifying the causes of injuries or incidents, but the main objective is to determine what went wrong and what actions are required for prevention or recurrence. Sensemaking is also delineated as a matter of action, with event assessment and post-assessment follow-on action being a cyclical process [8]. In accident investigations, corrective and preventive actions (CAPA) are developed as strategically planned actions to immediately correct the problems identified as the cause for the incident or injury, and to prevent recurrence. CAPAs are designed and implemented as a direct result of the root cause investigation findings.

\section{Senior Regulatory Quality Director review of root cause investigation}

The Senior Regulatory Quality Director, a senior compliance leader for the organization, is responsible for review and approval of root cause investigations for incidents that result in RI and LTI injuries. Upon initial review of the incident investigation report, there were no arguments or requests for changes in the submitted report. However, two months postsubmission, a second review was performed. Upon this review of the incident, the Senior Regulatory Quality Director read the entire report, and despite all of the information provided regarding the process, decided to edit and redefine the root cause, logging it in the system under a different determination than the investigators and root cause experts that were involved in the investigation had concluded. The root cause determination made by the Senior Regulatory Quality Director was that the materials were delivered in a hardened state. The justification for this change was that the "...vacuum hoist was used because raw materials were delivered in a hardened state and had to be massaged. If materials were delivered with normal consistency, the hoist would not have been used."

The logic behind this selection was that, although the employee was injured as a result of the head plate being stuck under pressure and the failure to prescribe troubleshooting, preventive maintenance, and training requirements, the 
hardened material is what caused the use of the vacuum hoist. Following that chain of reason, if the material was not delivered in a hardened state, it would not have to be placed on the shaker, and therefore the vacuum hoist would not have been needed and thus not used. As a result, the head plate would have never been stuck and the employee would have never been injured during the attempted removal of the vacuum-sealed head plate. Clearly, this determination is logical. It is true that the employee would not have been injured if the hoist was never used. However, this line of reasoning for root cause determination fails on multiple levels.

\section{Investigation team leader interaction with Senior Regulatory Quality Director regarding the root cause investigation determination}

Upon completion of the incident investigation and presentation of the report and summary, the Senior Regulatory Quality Director, who does not work on the manufacturing facility, criticized the report and requested that the investigation report be modified to list the hardened cement compound as the root cause for the injury. When the lead investigator and his team attempted to discuss and explain the scenario to the Senior Regulatory Quality Leader, they were met with a lack of attention and dismissal, followed by a stern argument defining the hardened cement drum root cause logic. When offered professional advice by a root cause investigation subject matter expert, the Senior Regulatory Quality Director became aggravated and loud, completely disregarding any advice provided, and increasing argumentative and reticent attitude. Furthermore, the Senior Regulatory Quality Director argued “I've been doing this for years, and I am paid way too much to be correcting you on this." Regardless how much the lead investigator attempted to collaborate and explain the situation, the Senior Regulatory Quality Director refused to listen to an attempted step-by-step explanation of the investigation or to consider options.

\section{Senior Regulatory Quality Director's interpretation and final assignment of CAPAs}

Upon completion of the root cause investigation review, CAPAs were assigned based on the Senior Regulatory Quality Director based on the modified root cause determination. The corrective action assigned called for the immediate removal of the vacuum hoist from use. The unit was required to be labeled "out of service" and a purchase order was placed to have the hoist repaired by the manufacturer. The preventive action focused on preventing the delivery and use of the hardened cement. The logistics manager was assigned an action to notify the supplier of the cement compound regarding hardened material and file a complaint, notifying the supplier that the company would no longer accept hardened product. Warehouse receiving staff were instructed not to accept the cement when it is delivered in a hardened state.

\section{Problems with the root cause and CAPA assignment}

The major problem with the re-assignment of the root cause by the Senior Regulatory Quality Leader was that addressing the cause could have prevented that particular injury during the situation, but was not the main point of cause for the problem. The moveable arm vacuum hoists in the warehouse were purchased to assist lifting and moving numerous items within the warehouse. The hoist is used for lifting materials other than the hardened cement drums. It is used for lifting and moving other heavy and awkwardly-shaped equipment, as well as 50 gallon drums of other compounds. The head plate could have become stuck on any other surface of materials being moved, and could have resulted in similar incidents. The removal of the need to shake the cement drum to loosen it from the hardened state did not preclude the problem.

The corrective and preventive actions assigned were only adequate to partially address the problem. The corrective action to immediately remove the single vacuum hoist and have it repaired would prevent the immediate problem with the one piece of equipment. However, numerous identical hoists exist in the facility, and could experience the same problem, since none of them were on a preventive maintenance schedule and not troubleshooting procedure existed for any of them. Furthermore, preventing the arrival of the hardened cement compound removed the necessity to lift and shake the drums of the specific compound. Nonetheless, the hoists are used for other processes throughout the facility. The re-assigned CAPAs failed to address these issues.

\section{Results and Discussion}

Evaluation of the Senior Regulatory Quality Director's sense making showed critical flaws that resulted in failed leadership and organizational ineffectiveness. The result of ineffective sensemaking by the senior organizational leader resulted in undesirable organizational implications, affecting suborganization teamwork. Additionally, had the situation been left to remain placid without intervention, the poor leadership decision could have resulted in unsafe working conditions and potential incident recurrence.

\section{Analysis of Senior Regulatory Quality Director Decision and Sensemaking}

There were numerous failures during the root cause investigation review process by the Senior Regulatory Quality Director that resulted in ineffective sensemaking and thus poor decision-making. These included problems with

i. perception

ii. limited search

iii. cognitive and confirmation bias

iv. noise

v. communication

vi. institutional factors

vii. power

viii. ego 

ix. collective knowledge
x. assessment of narrative
xi. problem-solving
xii. emotion
xiii. interpretation and
xiv. politics of knowledge.

\section{The effects of perception, interpretation, and perspective on sensemaking}

Perception of situations, including during root cause investigation, are very much guided by cues that are received from the environment as well as from experience. Sensemaking usually begins in a state of chaos [8], which is often exactly what is experienced at the front end of root cause investigations. This chaos is rife with an undifferentiated flux of fleeting sense impressions from all of the raw activities and information received through environmental and personal cues [8]. The perceptions and interpretations, couple with experience, communication, and other factors creates mental models that can also be shared to make meaning of situations. This is achieved through organizing, which is essentially partitioned and established through bracketing. Once the flux raw data and activities are available to the individual, sense making becomes about bracketing and labeling [8].

Bracketing is guided by mental models [8]. Bracketing is an incipient state of sensemaking that is based on a diversity of available data. Based on what has already been learned, past experience, and the information available, decisions can be made. These decisions are achieved through partitioning and organizing data from a combination of mental models and salient cues [8]. During this process, phenomena are forcibly carved from the flux of raw information. In the root cause scenario, this is one primary area where the leadership fails because the Senior Regulatory Quality Director identifies with raw information and experience and stops there, with limited information and a lack of analysis of all of the data and cues received. Essentially, bracketing is cut short, and though the world is simplified [8], it is too simple, leaving a lot of data on the table not to be considered, resulting in incomplete labeling and premature decisions. Sense making requires imposing functional labels on interdependent events to suggest plausible acts of management, coordination, and distribution [8]. Incomplete analysis and premature labeling can result in categorization using peripheral cases with equivocal meaning, with indeterminate actions more likely to alter sense making [8]. In root cause investigation, the limited bracketing and immediate labeling, using only peripheral cases of cause and contributing factors resulted in assumptions, leading to equivocal rather than empirical data.

Interpretation is also vital to sense making, and can affect the sense making process and the actionable results of the process. It can be said that no two people interpret things in exactly the same way. This is because meanings people develop are fundamentally fluid and they continue to develop over a breadth of experiences, making them essentially unstable and $[22,23]$. Some scholars contend that there is no unified or shared representation in organizations as a result of this because histories are too diverse and complex [21]. In addition to interpretation based on experience and the fluidity of experiences, individuals and their sense making abilities are also affected by cognitive and confirmation biases.

Psychologist argue that mistakes in our judgments cannot possibly be random or else they would cancel each other out (2011). Instead, they maintain that human judgments are systematically biased towards one side or the other. These are cognitive biases that we maintain as a result of biology and experience (2011). [19], explain the role of cognitive bias in human judgement, presenting that biases resulting from character based upon personality, created by factors including heredity, biology, and psychology. These include things like
a. biologically-limited information processing capacity
b. hermeneutics
c. social influences
d. emotional and moral motivations, and
e. noise.

One of the key elements identified in cognitive bias is noise and noisy information processing [19]. Besides the way that our brains process noisy information, Kahneman presents the concept of noise as environmental exposure that affects evershifting attitudes and perspectives of individuals because of the influence of unimportant or irrelevant things, including
a. the weather
b. dining menus and
c. traffic patterns among other topics.

this irrelevant data can affect mood or information processing, and thus result in a situation in which an identical question being asked multiple times over extended periods potentially results in different responses from the same individual [19]. These inconsistent decisions can dramatically affect organizations and organizational outcomes relative to mission and goals [19]. This noise is considered a type of cognitive bias, affecting sensemaking, and therefore decisionmaking.

This cognitive bias is evident in the root cause investigation scenario when the Senior Regulatory Quality Director did not immediately negate and modify the determination of the root cause, but re-reviewed and requested modification two months after initial submission and review. It appears that the senior leader was somehow refocused and potentially made a different determination after initially agreeing to the root cause investigation report during the initial review. Narrative 
devices are very useful in sense making. Narratives serve as a sort of detective story example in which the problem-solving procedures entail a set of movements and actions that detect the cause of the problem, identifying a diagnosis, and agreeing upon corrective actions [14].

As a narrative process, problems are dramatized and made visible and meaningful structures and cognitive frames are created, permitting diagnosis to occur by transforming equivocal findings into meaningful characterization [14]. Furthermore, narratives deal with human intentions, and their significance in the study of organizations have been recognized as the basic principle of human cognition $[14,24]$. The narrative method implies collecting data from varying sources and piecing information into a comprehensive and useable text. This method is what implicitly transpires during root cause investigations. Making sense of a thorough collection of data beyond the raw material is necessary and can lead to actionable intelligence.

The Senior Regulatory Quality Director failed to consider the entire narrative, thus limiting the amount of information from which to make root cause and CAPA decisions. Instead of thorough analysis and interpretation of a comprehensive data pool, the Regulatory Director relied on limited search, meaning that there was no attempt to look at alternatives. Narratives provide the fundamental vehicle for securing the commonsensical in organizational knowledge [14]. However, the Senior Regulatory Quality Director ignored the narrative provided in the investigation and created a narrative that best fit her biases. As soon as she noticed the hardened material as a contributing cause, the fixation was on that individual problem, with blatant negation of other causes, although they were provided. The Senior Regulatory Quality Director committed to the hardened cement drum as the cause. She identified the problem that was convenient and acceptable for her interpretation, and closed the door on other possibilities, leaving no room for doubt and re-evaluation.

Additionally, [5] also examines the potential influence of confirmation bias on sensemaking. The Senior Regulatory Quality Director had the full narrative and a comprehensive amount of information available for review. Nevertheless, she was readily able to select one individual contributing cause to the incident, attach her mind to it, and treat it without any doubt as the root cause. [5] describes that humans tend to seek out facts that support their ideas and beliefs, stopping short of any material that could be revealed to discredit them. Perhaps this is, in part, fueled by hubris. Not leaving room for doubt and reevaluation implies idea of perfection or inability to be incorrect. This is similar to [24] Reality Filtering, which is defined as “... many among the intelligentsia create their own reality- whether deliberately or not - by filtering out information contrary to their conception of how the world is or ought to be [24]." Furthermore, this can be interpreted as an example of withdrawn self dishonesty, an organizational behavior theory in which the individual making decisions is situationally withdrawn, due to a lack of self-awareness and ego, and is therefore unable to recognize his or her own role and participation in an organizational failure.

In this scenario, the Senior Regulatory Quality Director exhibited numerous signs of excessive pride and egotism. The blatant disregard shown for the investigation team and the subject matter experts, including direct refusal to engage in conversation, reduced the chances of comprehensive data gathering and complete understanding of the event. Action is the meaningful element in organizational sense making, but the construction of the action is subject to conflict of interpretation with the resolution firmly rooted in argumentation and debate [14]. Without the discourse, the entire sense making process potentially becomes affected and may lead to ineffective decisions, such as was experienced with the root cause investigation.

Sensemaking methodology is partially grounded in human perspectives [18]. Perspective has a great deal to do with decision-making, and evaluating and understanding different perspective in a given situation can be vital in making valid decisions. Organizational teams commonly struggle through conflict resulting from diversity of thought and experience. In order to avoid this, teams must evaluate diverse perspectives from within the group. Diversity means that team members have different experiences and ideas. These differences should be shared and explored. Diversity of thought and ideas are not weaknesses, but rather opportunities [25]. The idea behind sharing perspectives, also known as street corners, is focused on ensuring team members are open to sharing ideas and openly and honestly listening to the ideas of others to aid in the design of the best possible solutions to problems [25,26]. Dervin [6] exemplified this in her work, refining a methodology in this area by focusing on the communication of information and how that exchange can enhance or impede sensemaking [18].

This concept is critical to sensemaking and root cause investigation because sensemaking is not about truth and getting the exact correct answer. Sensemaking is about establishing the best and most comprehensive plausibility for a situation through continuous re-evaluation and re-drafting of the story and incorporation of all of the data as it becomes more available [8]. This is important because root cause investigations require a comprehensive assessment of all of the data points available, and root cause analysis tools are used to take into account as much data as possible to make root and contributing cause determinations. In the vacuum hoist analysis, the Senior Regulatory Quality Director failed to observe or consider all of the data or to communicate effectively with the root cause investigation team that participated in the investigation. The senior leader chose, instead, the make a determination based 
on experience and perception. However, the research shows that leaders' and managers' perceptions are rarely accurate $[8,20,27]$.

Organizational activity, managerial practices, and decisionmaking often rely heavily on the accuracy of leaders' and managers' perceptions [27]. Organizations and teams often make the assumption that managers and leaders have accurate perceptions of their organizations or their work environment. However, the research suggests that their perceptions are highly inaccurate [27]. Studies exhibit that perception is formed by the prior beliefs and needs of the perceiver rather than objective facts, which supports the necessity to collaborate and communicate with team-mates or co-investigators to secure a more comprehensive set of data on which to make decisions [20]. In fact, organizational behavior and organizational development experts propose that a significant and unfavorable problem for organizations does not have to do with inaccurately analyzing scarce data, but rather failing to interpret an abundance of data into something actionable [8]. Therefore, by ignoring the data presented by the investigation team, and refusing to discuss the situation with the subject matter experts from an ethnographic perspective, the Senior Regulatory Quality Director constrained the collective ability for the team to manage their information and prevented the group from collectively reaching a comprehensive conclusion and appropriate actions. Sense making requires the cognitive processes by which people engage in debate, dialect, and collective inquiry [14]. The senior leader failed to foster that, and that action, or lack thereof, contributed to failed sense making and bad decisions.

Furthermore, the Senior Regulatory Quality Director does not work on the main manufacturing site. Ethnography can be critical to root cause investigation. Being embedded in the environment where the incident occurred is often necessary and ideal for a thorough understanding of the processes involved in the workplace. Psychologist and ethnographers have exhibited through research that competence is not readily separable from situational artifacts, including physical environments and social relations [20,28]. Likewise, being present during the investigation, and having a clear understanding of the situation that transpired during the incident, and the perspectives of the people involved is important to determine root and contributing causes and factors. It is not uncommon, and is usually prudent practice to visit the site of the accident and even recreate the incident as it occurred. This is similar to forensic assessment during criminal investigations. In this scenario, the Senior Regulatory Quality Director did not work in the area and did not participate in the on-location investigation. This senior leader therefore had very limited understanding, based on limited historical experience, of the environment and the processes in the warehouse. The lack of detailed information, and the failure to evaluate details provided by the investigation team, resulted in a limitation on perspective. This type of limitation seriously affects the potential for effective sensemaking, and can be particularly dangerous when people's safety is at stake.

\section{The effects of institutional factors on sense making}

Research suggests that institutional context precedes and follows action, explaining that actors make sense within institutions, not outside of them or despite them. These institutional factors, including

a. position

b. authority

c. company policies and

d. hierarchical structure and

e. culture contribute to shaping what is also referred to as signification or meaning-making through interpretation $[29,30]$.

It is no surprise that people who are powerful and advantaged have unequal access to roles and positions that influence construction of social reality, including those that drive decisions [8]. When sense making involves group votes, power roles may affect whether the votes are weighted equally. Power can affect sense making because of control over

I. cues

II. discussions

III. criteria for plausible stories

IV. permitted actions

V. histories decided upon and

VI. valued or derogated identities [8].

It was evident during the root cause investigation review that institutional factors played a role in the outcome. The Senior Regulatory Quality Director behaved with hubris, not only making statements regarding having worked with root cause investigations for over thirty years, but adding that the expertise of the past thirty years was not to be questioned. The demand to modify the root cause and CAPAs in the incident investigation report was a power play, regardless of their correctness. When approached with recommendations based on professional advice from the ethnographic experience, the Senior Regulatory Quality Director shut the advance down immediately, telling the lead investigator in a loud and stern voice not to argue and to do what is instructed, followed by walking away from the conversation. This was a textbook display of the use of positional power and authority. The root cause lead was hired based upon

- leadership experience,

- $\quad$ root cause expertise, and

- straight forward and honest approach to communication and discourse.

However, when the opportunity to affect the process arose, the leader was not only ignored, but immediately silenced. Power in sensemaking can be expressed in many ways, including whether identities are valued or derogated [14]. In this 
situation, the position of the lead investigator and the status of the individual as a root cause analysis expert were not valued, and the actions taken by the Senior Regulatory Quality Director directly derogated. The senior leader exercised misuse of power and authority in order to control

- the discussion,

- who was involved in the discussion, and

- the overall decision based on personal preferences and cues.

This does not mean that it was done with any ill will, but was ineffective sensemaking nonetheless, identifying an incorrect root cause and deciding upon inadequate corrective and preventive actions. The position, authority, and hubris allowed the senior leader to assign blame for the incident to an entity that certainly contributed to the incident, but was not the cause for it. As a result, preventive measures would not have been implemented if the other causes were not addressed.

\section{Implications of the Senior Regulatory Quality Director's decision}

Sensemaking, as a subject, investigates two questions. The first question investigates what occurred in a given situation that led to a particular outcome. The second queries what to do about it. In other words, sensemaking is not just about organizing and figuring out what transpired in an event. It is about action [8]. Effective sensemaking in root cause investigation drives the direction of actions towards corrective and preventive measures relative to incidents and injuries. In the vacuum hoist injury investigation, the incorrect CAPA was finalized, not addressing the proper cause and therefore, not assigning adequate measures to prevent recurrence of the incident.

Furthermore, the team of investigators has witnessed the entire incident, and has experienced the non-constructive criticism and authoritarian approach regarding their work. As a result, they have lost confidence in their abilities to produce the necessary investigation product, and have lost the ability to make sense of what transpired during the incident review. They have limited understanding what the senior leaders want to see, particularly as a result of the noise in the scenario, and the disparate and nonsensical decisions. Furthermore, trust can be an issue in sense making. Trust is a key element that has been strongly connected to strategic partnership Adobor [18]. Trust is inherently needed in teams because when individuals in teams are interdependent on each other during work, they must be willing to risk reliance on each other in order to meet project deadlines. Thus, a culture of mutual trust is absolutely critical in supporting the core components of teamwork [31]. Trust is defined as the belief that no individual within an exchange will exploit the counterpart's vulnerabilities [18]. However, the breaking of trust, such as that perceived by the investigation team, can directly affect future relationships as well as the sensemaking process going forward. Investigators will have the notion or complex thoughts based on the experiences with the Senior Regulatory Quality Director every time they attempt to make root because determinations. The situation has created confusion and disarray in the root cause investigation process. As a side effect, the team has experienced a loss or reduction in their sense of confidence over team effectiveness. According to [19] sensemaking methodology, the Senior Regulatory Quality Director did not demonstrate effective leadership in this scenario. By not supporting the team and the process, a wedge was created around trust and value, affecting the relationship between staff, site business management, and senior management.

\section{Conclusion}

According to [14], organizational sensemaking is birthed on the trajectory that moves processes from equivocal to actionable, driving towards agreed-upon facts and epistemological closure [14]. Sensemaking is a critical piece of the toolbox necessary for leadership effectiveness in groups and organizations, enveloping key leadership objectives such as

a. Discussion,

b. Information exchange,

c. Comprehension,

d. Understanding,

e. Interpretation, and

f. Agreement when it comes to attempts to resolve problems [32].

This process is vital for effective decision-making in many facets of organizational operations, including injury and incident investigations. Root cause investigations for occupational safety incidents and injuries are akin to sensemaking in that they also originate in chaos from which raw information and data must be organized using root cause analysis tools. These tools are used in order to create a comprehensive narrative of the incident and all of the factors that contribute to incident or injury.

Our research took a phenomenological, grounded approach, performing a qualitative retrospective analysis using naturalistic observation during ethnographic positioning and formulating and evaluating a narrative of an injury investigation case study within a manufacturing plant. We analyzed a sensemaking process of senior leadership after the conclusion of a root cause investigation. Our findings exhibited the complete failure of organizational sense making on the part of a senior leader as a result of flawed
I. perception,
II. perspective,
III. narrative assessment,
IV. interpretation,
V. participation, and
VI. institutional factors. 


\section{Juniper Online Journal of Public Health}

The resistance of the senior leader to participate in discourse and comprehensive evaluation due to ego and power, and the selection of root cause based on limited search and confirmation bias, resulted in poor decision-making that could have had drastic negative effects on the organization and employees.

This case study serves as a solid example of sensemaking, describing and defining the importance and exhibiting not only where sense making can go wrong, but also the potential implications of inadequate sensemaking by individuals within organizations. There is considerable room for further case study in sense making in industrial settings and other organizations.

\section{Opportunities for Future Research}

Weber and Glyn [30]credit Weick's work as the cornerstone of organizational theory regarding sense making, but communicate that Weick's focuses on the micro-level approach regarding the process, expressing that additional study regarding the macrolevel is needed [30,31-35]. Macro-level research is not absent from the literature, but there is room for additional research in a diverse group of topics related to sensemaking, including a deeper dive into institutional factors and their interplay as a substantive part of sensemaking, arguing that institutions themselves serve as a primer for sensemaking [36-41]. Essentially, although there are volumes of research on theory and application of sensemaking, there is a dearth of case studies related to industrial environments and issues of work place safety [42-45]. Ample opportunities exist for further exploration of sense making application in industrial environments and the alignment of theory with case studies for organizational effectiveness and organizational safety culture [46-48].

\section{References}

1. Shufutinsky A, Shanahan P, Schaal N, Madad S, Johnson D (2015) Applying Conflict Analysis and Resolution Strategies to Assess Organizational Safety Culture in Accident Investigations. International Journal of Interdisciplinary and Multidisciplinary Studies 2(3): 71-90.

2. Abdelhamid TS, Everett JG (2000) Identifying Root Causes of Construction Accidents. Journal of Construction Engineering and Management 126(1): 52-60.

3. Carroll JS, Rudolph JW, Hatakenaka S (2002) Lessons learned from non-medicalindustries: root cause analysis as culture change at a chemical plant. Qual Saf Health Care 11(3): 266-269.

4. Iedema RAM, Jorm C, Long D (2006) Turning the medical gaze in upon itself: Root cause analysis and the investigation of clinical error. Soc Sci Med 62(7): 1605-1615

5. Kahneman D (2010) Thinking, fast and slow. New York, NY: Farrar, Straus and Giroux.

6. Dervin B (1998) Sense Making Theory and Practice: An Overview of User Interests in Knowledge Seeking and Use. Journal of Knowledge Management 2(2): 36-46.

7. Hilbert, M (2012) Toward a Synthesis of Cognitive Biases: How Noisy Information Processing Can Bias Human Decision Making. Psychol Bul 138(2): 211-237.

8. Weick KE, Sutcliffe KM, Obstfeld D (2005) Organizing and the process of sensemaking. Organization science, 16(4): 409-421.
9. Wick KE (1993) The collapse of sensemaking in organizations: The Mann Gulch disaster. Administrative science quarterly 38: 628-652.

10. Davidson JE, Daly BJ, Agan D, Brady NR, Higgins PA (2010) Facilitated sensemaking: a feasibility study for the provision of a family support program in the intensive care unit. Crit Care Nurs Q 33(2): 177-189.

11. Weick KE (1995) Sensemaking in Organizations.Thousand Oaks, CA Sage. Human Resource Development Quarterly 9(2): 198-201.

12. Weick KE (2001) Making Sense of the Organization. Oxford, Blackwell Publishers, England, UK.

13. Roy C, Andrews HA (1999) The Roy Adaptation Model. Stamford, CT: Appleton \& Lange.

14. Patriotta G (2003) Sensemaking on the Shop Floor: Narratives of Knowledge in Organizations. Journal of Management Studies 40(2): 349-375.

15. Weick KE (1977) Enactment Processes in Organizations. In Staw, BM and Salancik, G (Eds), New Directions in Organizational Behavior, Chicago.

16. Guiette A, Vandenbempt K (2013) Exploring team mental model dynamics during strategic change implementation in professional service organizations A sensemaking perspective. European Management Journal 31(6): 728-744.

17. Gioia DA, Thomas JB (1996) Identity, image, and issue interpretation: Sensemaking during strategic change in academia. Administrative science quarterly 41(3): 370-403.

18. Adobor $H$ (2005) Trust as sensemaking: the microdynamics of trust in interfirm alliances. Journal of Business Research 58(3): 330-337.

19. Kahneman D, Rosenfield AM, Gandhi L, Blaser T (2016) Noise. Harvard Business Review 39-50.

20.Winter SG (2003) Mistaken Perceptions: Cases and Consequences. British Journal of Management 14(1): 39-44.

21. Allard Poesi F (2005) The Paradox of Sensemaking in Organizational Analysis. Organization 12(2): 169-196.

22. Weick KE, Sutcliffe KM, Obstfeld D (2005) Organizing and the process of sensemaking. Organization science, 16(4): 409-421.

23. Boland RJ, Tenkasi RV (1995) Perspective making and perspective taking in communities of knowing. Organization Science 6(4): 350372 .

24. Sowell T (2009) Intellectuals and society. Basic Book, New York, USA.

25. Katz JH, Miller FA (2013) Opening Doors to Teamwork \& Collaboration 4 Keys That Change Everything. Berrett-Koehler Publishers, San Francisco, CA, USA.

26. Katz JH, Miller FA (2014) Learning from the Journey: OD Values. OD Practitioner 46(4).

27. Mezias JM, Starbucj WH (2003) Studying the Accuracy of Managers' Perceptions: A Research Odyssey. British Journal of Management 14(1): 3-17.

28. Hutchins E (1995) Cognition in the Wild. Cambridge, MA; MIT Press, UK.

29. Giddens A (1984) The constitution of society: Outline of the theory of structuration.

30. Weber K, Glyn MA (2006) Making sense of institutions. Organizational Studies 27(11): 1639-1660.

31. Salas E, Sims DE, Burke CS (2005) Is There a "Big Five" in Teamwork? Small Group Research 36(5): 555-599.

32. Yukl G (2013) Leadership in Organizations ( $8^{\text {th }}$ edn) MA: Pearson, 
Boston, USA.

33. Bureau of Labor Statistics. (2014). News Release: Employer-Reported Workplace Injuries and Illness Summary-2013, USDL-14-2183.

34. Carroll JS, Rudolph JW, Hatakenaka S (2002) Lessons learned from non-medicalindustries: root cause analysis as culture change at a chemical plant. Qual Saf Health Care 11(3): 266-269.

35. Davidson JE, Daly BJ, Agan D, Brady NR, Higgins PA (2010) Facilitated sensemaking: a feasibility study for the provision of a family support program in the intensive care unit. Crit Care Nurs Q 33(2): 177-189.

36. DiNardi SR (1997) The Occupational Environment - Its Evaluation and Control ( $1^{\text {st }}$ Ed.), Fairfax, VA: AIHA Press, USA.

37. Gordin DN, Polman JL, Pea RD (1994) The Climate Visualizer: Sensemaking through scientific visualization. Journal of Science Education and Technology 3(4): 203-226.

38. International Labour Organization (2011) World Statistic: The enormous burden of poor working conditions.International Labour Organization.

39. International Labour Organization. (2014) Safety and Health at Work A Vision for Sustainable Prevention. International Labour Office, Geneva, Switzerland.

40. Katz JH, Miller FA (2013b) Judging Others Has Not WorkedSo Let's Join Them. Executive Forum, Fall 70: 51-57.
41. Katz JH, Miller FA (2014) Learning from the Journey: OD Values. OD Practitioner 46(4).

42. Mandler G (1997) Human Nature Explored. Oxford, New York, USA.

43. Petersen D (1980) Analyzing Safety Performance, Garland STPM Press, New York, USA.

44. Riesman D, Reuel D, Glazer N (1950) The Lonely Crowd: A Study of the Changing American Character. New Haven, CT: Yale University Press, USA.

45. Rogers Y, Connelly K, Hazlewood W, Tedesco L (2010) Enhancing learning: a study of how mobile devices can facilitate sensemaking. Personal and Ubiquitous Computing 14(2): 111-124.

46. Roy C, Andrews HA (1999) The Roy Adaptation Model. Stamford, CT: Appleton \& Lange.

47. Shufutinsky A, Shanahan P, Schaal N, Madad S, Johnson D (2015) Applying Conflict Analysis and Resolution Strategies to Assess Organizational Safety Culture in Accident Investigations. International Journal of Interdisciplinary and Multidisciplinary Studies 2(3): 71-90.

48. Wilson MJ, Wilson ML (2013) A comparison of techniques for measuring sensemaking and learning within participant-generated summaries. Journal of the American Society for Information Science and Technology, 64(2): 291-306.

\section{Your next submission with Juniper Publishers will reach you the below assets}

- Quality Editorial service

- Swift Peer Review

- Reprints availability

- E-prints Service

- Manuscript Podcast for convenient understanding

- Global attainment for your research

- Manuscript accessibility in different formats

( Pdf, E-pub, Full Text, Audio)

- Unceasing customer service

Track the below URL for one-step submission https://juniperpublishers.com/online-submission.php 